\title{
The contradictions in the existing physics and the psychological factors that influence them to be valued
}

\author{
Runsheng Tu* \\ National Special Steel Quality Products Supervision and Inspection Centre, China \\ Received: 24 June 2020 \\ Revised: 02 August 2020 \\ Accepted: 03 August 2020 \\ *Correspondence: \\ Dr. Runsheng Tu, \\ E-mail: 2run3@sina.com \\ Copyright: $\odot$ the author(s), publisher and licensee Medip Academy. This is an open-access article distributed under \\ the terms of the Creative Commons Attribution Non-Commercial License, which permits unrestricted non-commercial \\ use, distribution, and reproduction in any medium, provided the original work is properly cited.
}

\begin{abstract}
Besides the limitation of cognition level, the factors that affect the correctness of academic evaluation are as follows: collusion-recognization effect, backfire effect, cognitive maladjustment, inattentional blindness and aesthetic fatigue. It's hard for readers (especially the reviewers of academic achievements) not to fall into the trap. Therefore, the probability of misjudgment of academic achievements of "questioning and denying current prevailing theories or creating new theories" is very high. The intolerable contradictions in the existing physical theories, such as space paradox, time paradox, quantum entanglement paradox, are exposed by means of thought experiments and "to present the facts, reason things out", and take these works as examples to reveal the formation and utilization of these adverse psychological effects. The conclusions are as follows: both special relativity and Copenhagen interpretation of quantum mechanics have fatal injury; it is difficult to eliminate the misjudgment of academic achievements, but the misjudgment rate can be reduced. The concepts of "invariance of spatial attribution" and "inaction association" are established. These works can urge readers to treat new and old physics theories more carefully, promote the development of physics, and promote the birth and improvement of the evaluation theory of science and technology.
\end{abstract}

Keywords: Relativity principle, Copenhagen interpretation, Quantum entanglement paradox, Collusion-recognization effect, Time paradox, Invariance of spatial attribution, Inaction association

\section{INTRODUCTION}

Jiqing published the serial blog "quantum mechanics stupid words" in "science net blog". ${ }^{1}$ There are also many scholars criticizing quantum mechanics. There are many other statements that question quantum mechanics. However, these voices of questioning have received little attention from mainstream scholars, and there only believe in non local-realism quantum mechanics. ${ }^{2,3}$ What's the reason? Anyone who knows a little bit of scientific knowledge (even for housewives) can point out one or two special relativity contradictions. There has been a lot of material questioning special relativity(and the material revealing the contradictory of relativity). Theory of relative-absoluteness can also be established. ${ }^{4}$ Why still believe in relativity? The answers to these two questions share common characteristics. The first is that the quality and level of the questioners are uneven. Most of the arguments in question are wrong. There are only a small proportion of strong doubts, and they have been submerged in a large pile of garbage and difficult to find. In addition, a popular theory is not $100 \%$ wrong, at least it can be applied to a certain range, and just like the wrong theories of the geocentric theory before the birth of Heliocentric theory can also guide people to observe and explain the movement of some celestial bodies. In addition to the above factors, the misjudgement of 
academic results by mainstream scholars is also an important factor.

There are many factors that affect the correctness of academic evaluation. The main factors are: interest factors, emotional factors, quality factors, environmental factors, cognitive limitations and learning factors. Correct views and theories grow and spread in the process of fighting against the thoughts and behaviours containing these factors.

This article focuses on the psychological factors that affect the accuracy of scientific and technological achievements. There are too many and too powerful factors to influence making a correct scientific evaluation. Even a serious scholar can hardly rule out the serious interference of these factors. No one can overestimate himself and promise that his comments on the voices questioning the existing theories will be absolutely correct even in another 50 years.

Physical theory generally goes through the period of eruption, prevalence, decline and replacement. This is the life course of theory. Even the most popular theories have contradictions and shortcomings (especially the theory of Physics). In other words, the theory of physics always develops in the periodic process of errors appearing and eliminating. That is to say, physical theory has to go through a process from birth to prevalence and then to decline. We think it's a law, and call it "the law of the rise and fall of theory". However, people generally do not believe that there are contradictions in the theories being used, especially the theory in the heyday. For example, during the period when geocentric theory prevailed, few people believed in Copernicus. At the beginning of the theory of relativity, few people believed it. This is the inertia of consciousness, idea or thinking. The determinants of this inertia can be divided into three broad categories. The first category is the no-fault factor (i.e., limited by the level of knowledge, also known as the limitation of knowledge. This is an objective factor). The second category is interest factors and emotional factors (including scientific beliefs). The third category of adverse psychological effects: "collusion effect", "nonattention blindness", "backfire effect", "cognitive dissonance" and "aesthetic fatigue".

The emergence and development of relativity and quantum mechanics (especially the Copenhagen interpretation of quantum mechanics) cannot escape the shackles of "the law of the rise and fall of theory". According to the "law of theoretical rise and fall", the next cycle of the development of relativity and quantum mechanics will definitely occur. That is, the decline of relativity and the Copenhagen interpretation of quantum mechanics are bound to occur.

We introduce space paradox, time paradox, quantum entanglement paradox (inaction-correlation paradox), and analyze the tactics of the defenders of the old theory.

\section{IMPORTANT INTERFERENCE FACTORS FOR THE ACCURACY OF SCIENTIFIC AND TECHNOLOGICAL EVALUATION, THE PSYCHOLOGICAL EFFECT OF "COLLUSION RECOGNITION" AND "INATTENTIONAL BLINDNESS"}

Misjudged events of new theories and perspectives occur in the development of each theory. Incidents of incorrect theories and opinions being misjudged as correct abound. There also are many opposite examples. Nobel laureate Honjo once said, I think $90 \%$ of the opinions in the magazines such as nature and science are not correct. Ten years after the publication of the paper, only $10 \%$ of the opinions can be considered correct. $^{5}$ A very famous academic journal has a very strict peer review system. Why does this happen? The reason is that there are "cognitive defects".

\section{INATTENTIONAL BLINDNESS}

There is a phenomenon of "inattentive blindness" in human observation and attention. This phenomenon refers to the fact that there are many contents or problems, but the people in front of them intentionally or unintentionally give a non comprehensive guidance observation and thinking opinion first, and the guided people can't see the contents or problems that are not in the guidance opinion. The psychological "inattentive blindness" is that when you concentrate on one problem, you tend to ignore another and easy to see, or should be very noticeable and should not be ignored. A common method of creating inattentional blindness is to steal the concept (another trick is to guide the reader away from the problematic place). Stealing concept is also a covert diversion of attention, so that the areas that should be concerned are not concerned. Inattentional blindness occurs.

For experiments verifying special relativity, they are generally only directed to focus on quantitative relativity effects. So, the qualitative verification of relativity principle is ignored. In this way, it is often said that "all experiments have proved the correctness of special relativity" is the prejudice caused by inattentive blindness. In fact, no experiment can directly verify the principle of Lorentz relativistic principle (that is, the perfect relativity of relativistic effect has not been verified).

\section{BACKFIRE EFFECT}

The backfire effect refers to: when a wrong message is corrected, if the corrected message is contrary to the original view, it will deepen people's trust in the wrong message. Many people have pointed out mistakes in relativity and the interpretation of quantum mechanics. However, most orthodox physicists think that the information of critical relativity and the interpretation of quantum mechanics are contrary to their original deep- 
rooted ideas. As a result, they believed more in relativity and existing interpretation of quantum mechanics. The resonance of cognition formed in this way is the result of the backfire effect, which can't prove that what people insist on is absolutely right. The history of human society (or physics) development is the history of fighting errors and prejudices. Often it is "it is not difficult to solve the problem, but it is more difficult to recognize the results (that is, it is more difficult to recognize the results than to research them)".

The backfire effect is similar to the anti-psychological psychology. Rebellious psychology is a mental state in which people take opposite attitudes, words and deeds in order to maintain self-esteem and face each other's requirements. The backfire effect is also a kind of rebellious psychological state. The difference is that the general counter psychology is that the weak resist the strong, and the counter fire effect is that the strong resist the weak.

\section{AESTHETIC FATIGUE AND COGNITIVE DISSONANCE}

"Aesthetic fatigue" was originally an aesthetic term. The specific manifestation is that the excitement to the aesthetic object is weakened, no longer has a strong sense of beauty, and even the object is disgusted. It now refers to the loss of interest in anyone or anything in life, and even the feeling of boredom, apathy or insensitivity.

The theory of cognitive dissonance was put forward by Leon Festinger. It means that individuals realize that there are contradictions between their attitudes or between attitudes and behaviours. The most famous example of cognitive dissonance is that in ancient China Women wrapped their feet. Knowing that it's harmful to our health, we all flock to it. It is thought that women's feet are small and beautiful, and even men do not marry non-three-inch Golden-Lotus.

\section{COLLUSION RECOGNITION EFFECT}

The so-called "collusion recognition phenomenon" (or "collusion recognition effect") refers to the belief that "leaders intentionally adhere to incorrect cognition, drive some people to follow the trend unintentionally, thus causing a wide range of resonance of incorrect cognition, and then generate thinking inertia and even do not argue right and wrong". The "leader" refers to the academic authority, influential people and powerful institutions. "collusion recognition" is very similar to "collusion up prices" (collusion recognition of the old theory is to coax up the value of the old theory).

According to the characteristics of the phenomenon, part of once the "collusion recognition phenomenon" is formed, there is "cognitive inertia". Passively collusion recognition is to recognize and support an opinion or theory in the form of "retreat from correct understanding and disregard the facts". For example, in the period of the prevalence of Aristotle's theory, people supported the idea that large objects fell faster than small ones, and in the period of Copernicus, people supported the geocentric theory, all of which were collusion recognition. There are many similar examples of negative textbooks. Because almost every theory has to go through a collusion recognition period (the prevailing period of theory is also its collusion period). Any theory of physics has to go through the following development process: forming period, prevailing period, declining period, disappearing or being replaced period. Now, Both the theory of relativity and the Copenhagen interpretation of quantum mechanics are in a period of decline and recognition.

No matter what kind of physics theory is understood by everyone. In the pyramid structure of the knowledge level, the next level always believes the previous level. In this way, academic authority can achieve "one call and one hundred responses". This laid the foundation for the collusion recognition effect. There are many famous collusion events. For example, during the Copernicus period, people relied on the geocentric theory transformed by Ptolemy and repelled the heliocentric theory; In the 20th century, the discovery of false N-Ray was highly sought after all over France.

The effects of "inattentional blindness", "backfire effect" and "cognitive dissonance" can enhance the "collusion recognition effect" and extend the "collusion recognition period" of the theory. Both theory and viewpoint have certain scope of application. In a certain period, the emergence of criticism of the old theory is normal and consistent with the laws of the rise and fall of the theory. Therefore, the most abhorrent aspect of collusionrecognition is not collusion-recognition itself, but rather a strong opposition to "the birth of new ideas and new theories and criticism of old theories." The inevitable manifestations of collusion are "pull-ups" and / or "inhibit the emergence and spread of new theories." This will obviously delay the development of science and technology. There are many examples in this regard.

\section{THE SPACE PARADOX AND TIME PARADOX CAUSED BY THE PRINCIPLE OF RELATIVITY}

There is a large rectangular rigid board with a hole of 6.6 meters long and 5 meters wide in A system. There is a relatively rectangular rigid plate (length 8.0 meters, width 4.8 meters, and thickness 0.01 meters) in B system. The small rigid plate moves obliquely to the hole on the large rigid plate at an angle of $45^{\circ}$ (Figure 1). The velocity component of the velocity of the small rigid plate in the plane parallel to the large rigid plate is $v_{\mathrm{x}}=0.6 \mathrm{c}$. According to the conclusion of the ruler contraction of the theory of relativity, observing in the A system, the length of the small rigid plate in the B system is 6.4 meters 
$\left(1 / \sqrt{1-v_{x}^{2} / c^{2}}=0.80, \quad 0.8 \times 8.0=6.4\right)$.

The thickness shrinks to 0.008 meters, and can pass through the hole in the A system. Observing in the B system, the length of the hole in the A system was reduced to 5.2 meters, and the small rigid-plate of 8.0meter-long could not pass through. Observers' conclusions in the two inertial frames are contradictory and cannot be overcome in the framework of relativity. For the sake of convenience, we call this kind of experiment "oblique via experiment", it is the rule of paradox (one kind of space paradox). It can be seen that space paradox exists in the special theory of relativity (important conclusion 1, IC 01 for short).

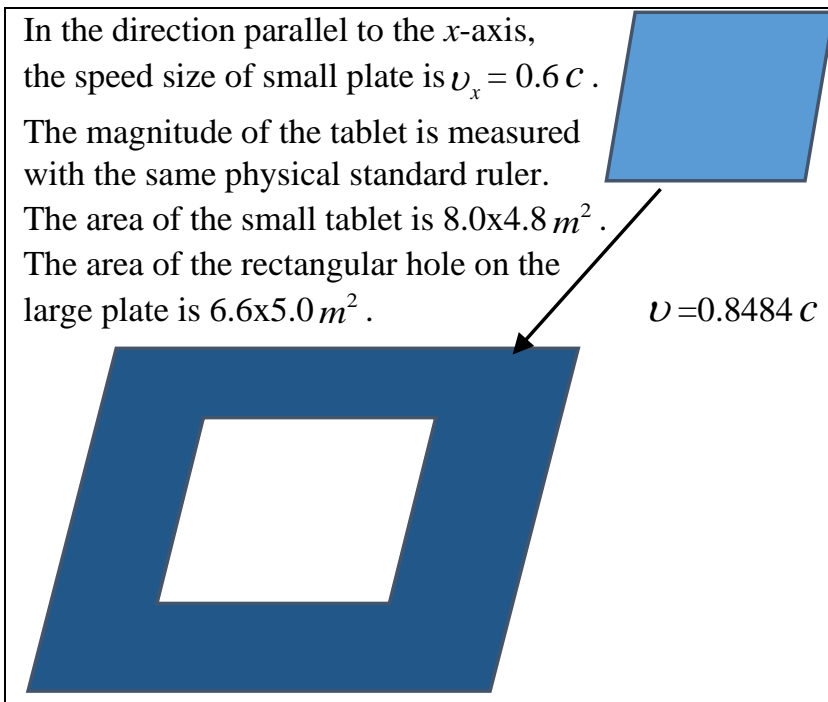

The relative motion between the upper and lower plates is a uniform linear motion. Observed on the large flat plate, the small plate above falls diagonally.

Figure 1: The space difficult. When observed on the large plate, the small plate can pass through the rectangular hole on the large plate, but observing the small flat plate, it doesn't. The law of refraction is broken.

If space contraction effect is visual effect, so is clock slow effect. The reason is that the wavelength and frequency of photons are related by the speed of light, and the standards of time and space (that is, standard clocks and standard rulers) are defined according to electromagnetic waves. "The clock slow effect is real, while the scale effect" is impossible depending on the effect. If the coordinate axis in the A system is rotated by 45 , the "horizontal moving plate passes through the hole in the horizontal direction" becomes the "horizontal movement inclined plate passes through the hole on the inclined surface". The quantitative calculation method of space contraction is exactly the same as above. We use uniform linear motion without acceleration, so we don't need to use general relativity. Therefore, we have no way to interpret this space problem as a false one. The space problem is a logical problem of the principle of relativity.

\section{INVARIANCE OF SPATIAL OWNERSHIP (BELONGINGNESS)}

Previously, it was thought that a vacuum was an empty space. The theory that the universe can and will create itself from nothing cannot be denied. Believing that there is an empty process at the beginning of the universe is conducive to establishing a theory of the universe from nothing. An empty space cannot interact with other substances. An object that does not interact with any space cannot move with the space it occupies, let alone with the space it does not occupy. The current view of vacuum is that vacuum is full of fleeting virtual particle pairs. The space filled with virtual particle pairs does not conform to the laws of thermodynamics. If the virtual particle pair is the space itself, then to make space move with the object, the moving object must exert force on the space (virtual particle pairs). Space can't be moved by the moving observer or object. In fact, as long as the space without any matter does not move, the centre of gravity of the virtual particle pair generated by such space points will not move. Otherwise, virtual particle pairs and their fields still only occupy space and cannot represent space itself. Even if there is a substance that can exert a force on the virtual particle pair, such an object cannot move with space.

As mentioned above, the space of infinity that cannot interact with material forces can only be an independent space, not a space where its belongingness (ownership) can be changed involuntarily. In other words, the reality, objectivity, and infinity of infinite space determine the independence of space. It is very difficult to change its affiliation. The movement of an object occupying space can only indicate that the position of the object in the space has changed, and it cannot represent that the ownership of the space itself has changed. The ownership of space does not change with the movement of the observer. This is called the invariance of spatial ownership, which is referred to as the invariance of spatial ownership (IC 02). Each part in the infinite space will not change the belongingness because the observer changes the state of motion. The change in the ownership of space in the principle of relativity can only be the intuitive vision of space. "Space invariance" denies the principle of relativity (that is, the soil on which the covariance of the laws of physics depends has been removed). According to the "invariance of spatial attribution", it can be predicted that there are other contradictions in the principle of relativity, and no spacetime relativity experiment can directly verify the principle of special relativity.

The core ideas of the above discussion are: Infinite space cannot move (its movement is meaningless), it can only be absolutely stationary; No one can apply force to space, and space can't have extra movement without force as 
much as objects; what is not dragged by anything is absolutely stationary; virtual particles and their fields can only occupy space, not space itself. The most appropriate ownership for infinite space is the absolute stationary system. This is also Newton's concept of space. Newton's mistake was just to look at the visual space associated with low-speed moving objects as an approximately absolute static space. The maintainers of relativity also believe that Newton's concept of space is wrong not to consider space variable. In fact, denying the principle of relativity cannot deny that the moving object contracts and the moving clock slows down. ${ }^{4}$ Within the framework of the special theory of relativity, the objectivity, reality and the properties that cannot interact with other objects deny the relativity of space (including the relativity of the belongingness of space and the relativity of the size of space). This is a fundamental space problem for special theory of relativity (Different expressions of IC 01).

We can deny the relativity of space ownership (or the variability of spatial ownership) by observing the generation of virtual particle pairs. Inertial system A has a ruler with a scale. There is one observer in each of inertial system B and inertial system C. The observer in system A observed a pair of virtual particles to be forming near the scale 0 of the ruler. The centre of gravity (centre of mass) of the pair of virtual particles is stationary relative to the ruler. The observers in B-system and C-system simultaneously observed that the pair of virtual particles were produced by vacuum points. Is the phenomenon they observe "the centre of gravity (centre of mass) of the virtual particles is stationary with respect to the ruler" or moving with respect to the ruler? According to the special theory of relativity, B-observer and C-observer must think that the centre of gravity of the pair of virtual particles moves relative to the ruler. The Lorentz transformation cannot transform two relatively stationary objects into two relatively moving objects (IC 02). Intuitively, even if the observer observes in different inertial frames, he cannot change the two relatively static points into two relatively moving points. It can be seen that the ownership of space changes with the observer's state of motion, and the space of motion cannot move with observer. It is also a space paradox of relativity to reveal the immutability of space ownership.

\section{REVEALING TIME PROBLEMS WITH THE METHOD COMPARING THE FAST-SLOW OF THE CLOCKS IN THE FORM OF DOUBLE SPIRAL}

The experiment of atomic clocks flying round the world in two-way proves that the clock slowing effect is not relative. Only standing in an approximate inertial system associated with a non rotating solar system can relativism be used to explain it. This interpretation is a sophistry. This justification has the following problems: First, it was not anticipated that a system connected to a non-rotating solar system was needed before the experiment began;
Second, when using the approximate inertial system, there is no uniform quantitative standard for the degree of approximation (how small is the acceleration of a system to be an approximate inertial system? They cannot answer this question). In addition to the experiment of "atomic clocks flying round the world in two-way", other experiments that verify the special theory of relativity can be observed on the surface of the earth (that is, the surface of the earth can be used as an approximate inertial system). Which acceleration system can be used as an approximate inertial system is subjectively determined only by the relativity maintainer as needed. This is difficult to get rid of sophistry, and logically cannot withstand scrutiny. In fact, thought experiments that reveal time problems can be designed into the form that can avoid the effects of accelerated motion (IC 03).

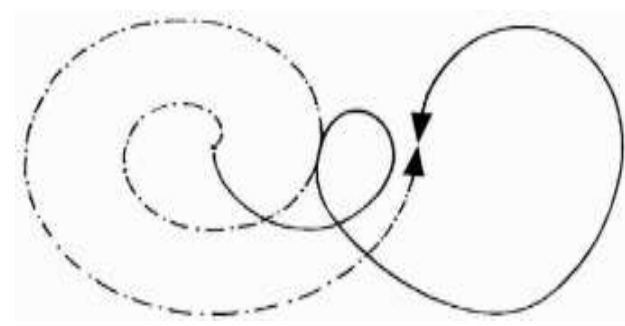

Figure 2: Method for checking clocks in double-spiral manner. Observe the two clocks that meet before and after in the inertial system. The order of whoever is fast is slow is definite.

In reference 4 , the method of collation clocks for a double helix is designed. ${ }^{4}$ When the clocks $\mathrm{A}$ and $\mathrm{B}$ in the two inertial systems of $\mathrm{A}$ and $\mathrm{B}$ meet for the first time, calibrate them, and then let the two clocks start to move in involute from the original stationary point. In this way, observing in a suitable third-party inertial system, when the two inertial systems A and B separate at an appropriate speed, the two clocks can meet again (Figure 2). Compare the time readings of these two clocks when they meet for the second time. If the principle of relativity in which each other's clocks become slower is true, then observer $\mathrm{A}$ and observer $\mathrm{B}$ cannot agree on which of these two clocks is faster. Because, in the case described, mutual observation is that each other's clock has one more degree of freedom of translational motion, and according to the principle of relativity, it can be concluded that the clock of the other party becomes slower. However, the fast and slow sequence of the clocks collated by the two encounters (meeting) of the two clocks is unique.

\section{A COMMON WAY FOR DEFENDERS OF RELATIVITY AND QUANTUM MECHANICS TO COVER UP OLD THEORIES}

The second section of this paper reveals the internal contradictions of the principle of relativity. The defenders of relativity will inevitably cover up these contradictions. They continue to evaluate new ideas on the basis of old theories. Their idiomatic trick is not to face these 
contradictions directly, let alone to justify them logically. Their approach has become a social law that is difficult to break. Planck's laws of science are part of it.

In the field of science, for the rejection of original theory due to its subversion, Max planck (April 23, 1858October 4, 1947, physicist, Nobel prize winner in physics), the founder of quantum concept and a famous German physicist, once had a famous saying: "A new scientific truth does not triumph by convincing its opponents and making them see the light, but rather because its opponents eventually die, and a new generation grows up that is familiar with it." This passage was later called "Planck's law of science". It vividly reflects the difficulty of accepting an innovation that subverts the existing system. The authority of participating coaxing recognition will not give up the theory of the coaxing recognition until they die. The victory of the new theory needs a long wait.

Physicists are conservative because of collusion recognition. For them, to abandon the old theory and innovate is a helpless choice after being driven mad by the experimental results. One example is the behaviour of Planck, the father of quantum. He put forward the quantization of energy out of helplessness, and then for many years he vigorously denied the quantization which was inconsistent with the classical theory. Experimental results that are inconsistent with the theory at the time are inherently difficult to find. Even if it is fortunately discovered, we will go through a long period of vigorous defence at the beginning of the discovery. You can see how difficult it is to make exceptions to Planck's Law. However, we must rely on these exceptions to speed up the pace of scientific development.

When encountering Lorentz's principle of relativity is inconsistent with the facts, the maintainers of the theory of relativity use "cannot stand in the non-inertial system to observe" to prevaricate. In fact, this reason is also problematic. The reason is that if we cannot observe in the strict inertial system, it is impossible for humans to have practical and meaningful observations on the verification experiments on the principle of Lorentz's relativity. (i.e., all experiments that test special relativity will be meaningless, because there is no strict inertial system in the experimental environment that humans can manipulate). If we pay attention to strict logic, relativity does not solve the problem of time paradox (twin paradox).

Some people may ask: Since the Lorentz principle of relativity cannot be universally established, why is Galileo's principle of relativity valid? My answer is: Galileo's principle of relativity does not take into account that mass changes with motion, and can only be approximately true. If Galileo's relativity principle is extended to Lorentz's relativity principle, its applicability will be worse. Many people say that "all experiments have verified relativity and quantum mechanics." In fact, it is the inattentional blindness effect that plays a role. Once this blindness is overcome, it will soon be found that many experiments do not support the Copenhagen interpretation of relativity and quantum mechanics.

Too many people find contradictions in relativity. There are many examples of inherent contradictions in the special theory of relativity. This shows that the theory of relativity should enter a decline period. However, the theory of relativity is still the official theory (orthodox theory), and academics cannot tolerate objections. This is because it is still in a period of collusion recognition. Now, special theory of relativity is just that authority is using their voice to maintain, not logic or reason. The establishment of authority and the acquisition of discourse power have established theoretical background. At the end of the development of an old theory, the discourse power of the authority of the old theory is based on the old theory. Once the authorities of the old theories admit the new ideas and theories, they lose the right to defend the old theories. If the authority of the old theory does not want to give up the right of discourse, it can only oppose the new theory and maintain the old one. Hawking, for example, was famous (establishing authority and gaining the right to speak) for his theory of relativity. Once he denies the theory of relativity, he has given up the right of speech. In fact, it's a small performance of character. If a physicist can deny the old theory and admit the new one in time, it just shows his greatness.

Defenders of orthodox theory often go into a logical cycle without knowing themselves. For example, Schrödinger cat reveals a contradiction in quantum mechanics. According to the original rules, this contradiction cannot be resolved, and they said that the Schrödinger cat state is the normal state of quantum mechanics. Einstein demonstrated the EPR paradox, and they said that "spooky action at a distance" is permissible and normal in the microcosm. In fact, in the macro world, Schrodinger cat state is not allowed to exist. The micro particles associated with it cannot be in the superposition state of decay and non decay.

\section{QUANTUM ENTANGLEMENT BELONGS TO THE SUBJECTIVE EXISTENCE OF "I SAW IT BECAUSE I BELIEVED IT"}

If the twin particles are not in the state of superposition before the measurement or the principle of superposition, the relationship between twin particles is equivalent to the relationship between a pair of gloves, not quantum entanglement. Only when we firmly believe that "the principle of superposition cannot be questioned" can the relevant experimental phenomenon be interpreted as quantum entanglement. It can be seen that the phenomenon of quantum entanglement belongs to the subjective existence of "I saw it because I believed it" rather than the objective existence of "I believe because I saw" (Because of the belief that "the principle of state superposition cannot be questioned", the related experimental phenomenon is regarded as quantum entanglement). From an experimental point of view, the principle of state superposition benefits from an 
explanation of the double-slit diffraction experiment. However, there are many alternative explanations for the double-slit diffraction experiment. In this way, it is simply too loose to elevate the quantum state superposition hypothesis to the principle. Besides, no one can observe the quantum superposition state. Obviously, the principle of state superposition is not beyond doubt, and quantum entanglement is not beyond doubt. The question of quantum entanglement is like a wave, rising higher and higher. ${ }^{6-19}$ Quantum entanglement also has the paradox of "spooky action at a distance" (also called inaction correlation paradox).

\section{CONCLUSION}

Science is constantly advancing, and the level of human cognition cannot reach its peak. There are too many traps for scientific and technological evaluation, and it is difficult for the most serious scientists to completely avoid the trick. If it is effective enough to overcome the adverse psychological effects of the scientific evaluation described in this article, it will be admitted that there is an insurmountable logical contradiction in theory of relativity and the Copenhagen interpretation of quantum mechanics. If theory of relativity is wrong, why can experiments prove the relativistic effect? The reason is that the correct theory is relative-absolute theory (theory of relativity absoluteness). In the theory of relativity absoluteness, the clock of absolute motion slows down and the object of absolute motion contracts. The surface of the earth can be used as an approximate absolute stationary system in most experiments. In addition, the Lorentz transformation is established in electromagnetics, as well as in absolute static systems. In short, developing relativity into relative absolute theory can eliminate many contradictions in relativity. The situation of quantum mechanics is similar. By abolishing Copenhagen interpretation, we can establish a quantum mechanical interpretation system of localized realism, and the mathematical form system of quantum mechanics is basically unchanged. Scienology is a comprehensive emerging discipline that studies the development laws of science and scientific activities and their social functions. Exploring the laws of scientific development first involves how to view and evaluate the scientific theories of the past and present (Especially what new views and theories are given to support). It can be said that there is no science without correct scientific evaluation. Therefore, the studies of science-technology evaluation are an important part of scienology.

\section{Funding: No funding sources}

Conflict of interest: None declared

Ethical approval: Not required

\section{REFERENCES}

1. Jiqing Z. Quantum mechanical nonsense. Science Blog, 2019. Available at: http://blog.sciencenet. $\mathrm{cn} /$ home.php? $\mathrm{mod}=$ space\&uid $=52021$. Accessed on 3 June 2020.

2. Tu R. Quantum Mechanics' Return to Local
Realism. Newcastle upon Tyne: Cambridge Scholars, Publishing; 2018:1-290.

3. Tu R. Localized realism quantum mechanics was born successfully. Beau Bassin: Golden Light Academic Publishing; 2019:1-313.

4. Tu R. Sound the Clarion Call Scientific Revolution. Beau Bassin: Golden Light Academic Publishing, 2019:1-538.

5. Honjo T. Don't believe what is written in the paper, $90 \%$ of the opinions in the magazines of Nature and Science are incorrect. GeenMedical, 2019. Available at: http://www.sohu.com/ a/363257056_750125. Accessed on 3 June 2020.

6. Einstein A, Podolsky B, Rosen N. Can QuantumMechanical Description of Physical Reality Be Considered Complete?, Physical Review. 1935;47:777-80.

7. Brunner N, Cavalcanti D, Pironio S, Valerio Scarani, Stephanie Wehner. Bell nonlocality. Rev Mod Phys. 2014.

8. Bohm D. A Suggested Interpretation of the Quantum Theory in Terms of 'Hidden' Variables. I. Phys Rev. 1952;85:166.

9. Freedman SJ, Clauser JF. Experimental Test of Local Hidden-Variable Theories. Phys Rev Lett. 1972;28:938.

10. Bell JS. On the Einstein Poldolsky Rosen paradox. Physics. 1964;1:195.

11. Aspect A, Dalibard J, Roger G. Experimental Test of Bell's Inequalities Using Time- Varying Analyzers. Phys Rev Lett. 1982;49:1804.

12. Leggett A. Aspect experiment, Compendium of Quantum Physics. Springer. 2014.

13. The BIG Bell Test Collaboration. Challenging local realism with human choices. Nature. 2018;557:2126.

14. Huang YF, Liu BH, Peng L, Li YH, Li L, Li CF, et al. Experimental generation of an eight-photon Greenberger-Horne-Zeilinger state, Nature Communication. 2011;546.

15. Bokulich A, Jaeger G. Philosophy of Quantum Information and Entanglement, 1-277. New York: University Cambridge Press. 2010.

16. Hensen B, Bernien H, Dréau AE, Reiserer A, Kalb $\mathrm{N}$, Blok MS, et al. Hanson. Loophole-free Bell inequality violation using electron spins separated by 1.3 kilometres. Nature. 2015;526:682-6.

17. Ejiang D. Syllogism of quantum mechanics, Science Blog. 2019. Available at: http://blog.sciencenet. $\mathrm{cn} /$ home.php? $\mathrm{mod}=$ space $\&$ uid $=3395754$. Accessed on 3 June 2020.

18. Lei Y. The world of quantum entanglement is unrecognizable. Science Blog, 2020. Available at: http://blog.sciencenet.cn/home.php?mod=space\&uid $=268546$. Accessed on 3 June 2020.

19. Tu R. If the wave function Collapse absolutely in the Interaction, how can the weird nature of particles are born in the interaction? - A Discussion on Quantum Entanglement Experiments. Indian J Sci Tech. 2019;12:1-10.

Cite this article as: $\mathrm{Tu} \mathrm{R}$. The contradictions in the existing physics and the psychological factors that influence them to be valued. Int J Sci Rep 2020;6(10):418-24. 